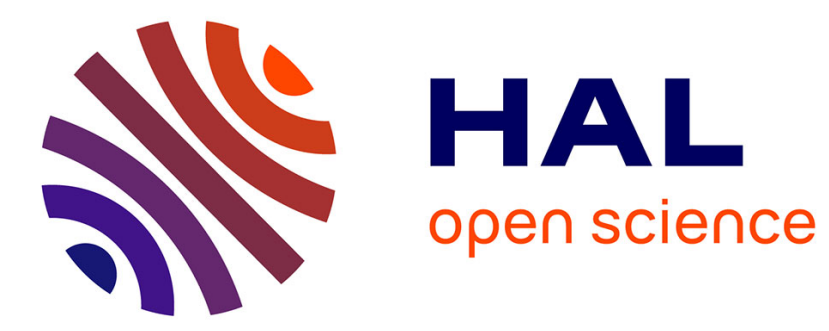

\title{
USAGES PROFESSIONNELS DE LA CATÉGORISATION ETHNIQUE EN ZONE D'ÉDUCATION PRIORITAIRE
}

Stéphane Zéphir

\section{- To cite this version:}

Stéphane Zéphir. USAGES PROFESSIONNELS DE LA CATÉGORISATION ETHNIQUE EN ZONE D'ÉDUCATION PRIORITAIRE. Migrations Société, 2010. halshs-01509018

\section{HAL Id: halshs-01509018 \\ https://shs.hal.science/halshs-01509018}

Submitted on 15 Apr 2017

HAL is a multi-disciplinary open access archive for the deposit and dissemination of scientific research documents, whether they are published or not. The documents may come from teaching and research institutions in France or abroad, or from public or private research centers.
L'archive ouverte pluridisciplinaire HAL, est destinée au dépôt et à la diffusion de documents scientifiques de niveau recherche, publiés ou non, émanant des établissements d'enseignement et de recherche français ou étrangers, des laboratoires publics ou privés. 


\section{USAGES PROFESSIONNELS DE LA CATÉGORISATION ETHNIQUE EN ZONE D'ÉDUCATION PRIORITAIRE}

Stéphane Zéphir

Centre d'information et d'études sur les migrations internationales | « Migrations Société »

2010/5 N 131 | pages 215 à 230

ISSN 0995-7367

Article disponible en ligne à l'adresse :

http://www.cairn.info/revue-migrations-societe-2010-5-page-215.htm

Pour citer cet article :

Stéphane Zéphir, « Usages professionnels de la catégorisation ethnique en Zone d'Éducation Prioritaire », Migrations Société 2010/5 (N¹31), p. 215-230.

Distribution électronique Cairn.info pour Centre d'information et d'études sur les migrations internationales.

(c) Centre d'information et d'études sur les migrations internationales. Tous droits réservés pour tous pays.

La reproduction ou représentation de cet article, notamment par photocopie, n'est autorisée que dans les limites des conditions générales d'utilisation du site ou, le cas échéant, des conditions générales de la licence souscrite par votre établissement. Toute autre reproduction ou représentation, en tout ou partie, sous quelque forme et de quelque manière que ce soit, est interdite sauf accord préalable et écrit de l'éditeur, en dehors des cas prévus par la législation en vigueur en France. Il est précisé que son stockage dans une base de données est également interdit. 


\section{USAGES PROFESSIONNELS DE LA CATÉGORISATION ETHNIQUE EN ZONE D'ÉDUCATION PRIORITAIRE}

Stéphane ZÉPHIR *

Cette contribution porte sur la mise en œuvre ordinaire de la "catégorisation ethnique" dans le cadre scolaire entre acteurs éducatifs, telle qu'elle se laisse analyser à partir de scènes observées. Nous montrerons comment les professionnels de l'éducation construisent collectivement une description de la situation scolaire des élèves et s'entendent, au cours même de leur discussion, sur des dispositions à prendre en fonction de caractéristiques spécifiques attribuées aux collégiens.

L'analyse présentée ici repose sur des données recueillies durant plusieurs années dans une zone d'éducation prioritaire (ZEP), dans la proche périphérie d'une grande ville du sud de la France'. Nous avons cherché à saisir comment les acteurs éducatifs construisent leurs ententes à travers leurs échanges langagiers en situation ${ }^{2}$. Cette démarche a nécessité un long travail d'approche des acteurs de terrain au cours duquel une relation de confiance ou d'estime réciproque s'est progressivement installée. C'est à ce prix que nous avons pu assister avec notre enregistreur à des situations d'interaction généralement fermées aux personnes statutairement étrangères au monde scolaire.

Nous présenterons ici deux situations différentes dans lesquelles les acteurs scolaires se rendent mutuellement observables et rendent compréhensibles les difficultés qu'ils rencontrent dans le cadre de leurs activités professionnelles. Bien que temporellement et spatialement distinctes, ces scènes forment un tableau de ce qui se joue de manière récurrente dans le cadre scolaire de la ZEP. L'arrière-plan normatif est un consensus professionnel relatif aux spécificités caractérisées comme ethniques de

* Docteur en sociologie, Université de Nice.

1. Cf. ZÉPHIR, Stéphane, Des différentes modalités de l'expérience minoritaire dans l'espace urbain d'une zone d'éducation prioritaire: les effets paradoxaux d'une action positive, thèse de doctorat en sociologie, Université de Nice, 2007, 540 p.

2. Selon une méthode microsociologique inspirée de Harold Garfinkel. Voir GARFINKEL, Harold, Studies in ethnomethodology, Englewood Cliffs : Prentice Hall, 1967, 288 p. 
certains élèves (première séquence analysée). II débouche sur des solutions supposées à la hauteur des difficultés spécifiques rencontrées par les acteurs avec ce type d'élèves dont les caractéristiques morales et comportementales sont dès lors naturalisées (deuxième séquence analysée).

\section{Un consensus professionnel sur la catégorisation ethno- culturelle}

La séquence présentée ci-dessous est issue d'une discussion entre les acteurs scolaires au sujet de l'organisation de la semaine Initiatives citoyennes. Dans ce passage, la documentaliste ouvre le débat en faisant référence à ce qu'elle nomme la violence des élèves. Elle illustre son propos en citant l'exemple d'un élève qui a administré un coup de pied aux fesses d'une camarade qui s'avéra être sa sœur.

E 1, E 4 : enseignantes de sciences de la vie et de la terre (SVT)

E 2 : enseignante d'anglais

E 3, E 5 : enseignantes de français

E 6 : enseignante d'histoire-géographie en classes de $4^{\mathrm{e}}$ et de $3^{\mathrm{e}}$

E 7 : enseignant d'histoire-géographie en classes de $6^{\mathrm{e}}$ et de $5^{\mathrm{e}}$

Documentaliste : Mais ils sont d'une violence absolument épouvantable ! Moi, ça me rend malade. Hier, dans les couloirs, alors qu'ils descendaient, j'ai vu un élève remonter l'escalier pour donner un coup de pied aux fesses d'une fille, mais alors avec une violence inouie ! Lorsque j'ai vu cela, je l'ai rattrapé pour lui demander s'il ne mesurait pas la gravité de ce qu'il venait de faire. Comment est-ce qu'il pouvait frapper une fille comme cela? Ça ne se fait pas, enfin ! II m'a répondu : " Ah, mais Madame, c'est ma sœur ! "I. Ils ont une manière d'agir avec les filles, mais alors !

E 1 : Ah, mais ils agissent de la même façon que lorsqu'ils sont chez eux ! C'est dans leur culture. Les filles sont au service des garçons.

E 2 : C'est comme cela qu'Abdoul Jabar agit avec les filles. Chez lui, c'est son frère aîné. Ici, comme c'est lui le plus âgé des garçons, il le remplace.

Documentaliste : Mais celui qui a donné un coup de pied à sa sœur, c'est absolument incroyable ! Et la jeune fille, elle n'a rien dit.

E 3 : Eh ovi, ils ont tous les droits. 
Documentaliste : Mais c'était d'une violence, avec des grosses chaussures ! Mais il y a tellement de choses à corriger. On l'observe, par exemple avec les carnets de correspondance. Quand on les leur donne, ils nous les arrachent des mains. On a beau leur dire : " Doucement, prenez les carnets délicatement "); ça ne change rien.

E 5 : lls crachent dans les plateaux.

Documentaliste : Ils crachent dans les plateaux ! C'est pas possible !

E 5 : En classe, au lieu de nous dire quelque chose normalement, ils hurlent. Mais c'est parce que eux ne sont pas habitués à la maison à parler sur un ton normal. Alors, nous, au bout d'un moment, on est agressé. Même avec la grande patience, au bout d'un moment, on est agressé.

Documentaliste : Et puis on voit aussi la crainte des filles. Dès qu'un garçon les approche dans les couloirs, elles se mettent à hurler, mais c'est un réflexe. C'est comme un réflexe, mais elles hurlent.

E 2 : De toute façon, ils ne se sentent pas français. En début d'année, en cours d'anglais, on fait Of what nationality are you? Moi, je leur dis toujours : "Tout le monde est français ". Et tu ne peux pas non plus les empêcher de le dire, ils sont les deux. Mais la première réponse qui vient à leur esprit, et ils ne connaissent que le mot French, ils ne connaissent pas les autres, eh ben, ils le disent à la française, mais ils disent d'abord "Tunisien " ou " Marocain ".

E 7 : J'irai encore plus loin : ils ne disent pas "Tunisien ", ils disent " Arabe ". lls ont déjà du mal à faire la différence.

E 2 : Alors que nous, en tant que professeur, on leur dit que tout le monde est français. Qu'ici, on est à l'école française, on est en France, donc que tout le monde est français. Ils n'ont pas de couleur, ils sont français.

E 3 : Ils sont déracinés.

E 2 : Et je pense aussi qu'il y a des notions très simples qu'on arrive difficilement à faire passer : c'est l'égalité entre les filles et les garçons. J'ai fait en classe avec les troisièmes des textes là-dessus, puisqu'ils existaient, et on a étudié le Code civil espagnol pour tout ce qui concerne l'égalité, etc. On a comparé avec le Code civil français, mais on a tout le temps des réflexions extrêmement désagréables sur : " Oh, eh ben non! Mais c'est pas vrai !". Mais je crois qu'il faut quand même le faire et continuer à le faire.

E 5 : Mais ils viennent de familles arabes et ils continuent à garder les mêmes manières de penser. Dans les familles, chez elles, les femmes sont dans leur coin, les hommes dans un autre. Il y a toujours ce même clivage.

E 3 : lls vivent une ambiguité.

E 2 : lls sont entre les deux, ils sont entre deux situations. 
E 5 : Dans leurs familles, ils vivent encore comme ça.

Tentons de dégager les composantes du consensus professionnel qui s'atteste progressivement dans cet échange.

\section{- Citoyenneté, violence, ethnicité : l'enchaînement discursif}

La prise de parole de la documentaliste inscrit directement le débat sur un registre qui va permettre l'organisation de cet enchainement discursif. La locutrice établit d'emblée une relation entre le thème de la discussion, les Initiatives citoyennes, et la violence des élèves. Dès que la conversation est entamée elle dénonce de manière hyperbolique ce qu'elle nomme la violence des élèves ("I Mais ils sont d'une violence absolument épouvantable ! Moi, ça me rend malade ") : on peut d'ores et déjà noter le caractère généralisant de son affirmation. La locutrice commence par avancer une proposition générale qui semble concerner l'ensemble des élèves. Ce n'est que dans un second temps qu'elle va illustrer son propos en décrivant une situation précise qui met en scène un élève particulier.

La scène décrite en apparence sous ses aspects purement factuels recèle les éléments favorables à une mise en récit qui se prête à une évaluation négative de l'ensemble des élèves garçons. L'opposition de genre filles/garçons va permettre de requalifier la scène par un saut qualitatif du geste brutal d'un élève envers sa camarade à une manière d'être inscrite dans un comportement culturel essentialisé. L'identification de la jeune fille comme la sœur de l'agresseur permet l'effectuation naturelle de ce passage qualitatif entre un acte d'indiscipline et un fait culturel stigmatisé par l'ensemble des locuteurs.

Le récit rapportant le coup de pied se trouve en effet inséré entre la première généralisation (IIIs sont d'une violence absolument épouvantable ! ") et une remontée en généralité tout aussi inclusive ("Ils ont une manière d'agir avec les filles, mais alors "I). À nouveau, la locutrice utilise le pronom personnel "ils" pour désigner non pas l'élève, mais une population de collégiens dans sa globalité.

Si généralisation il y $a$, cependant, celle-ci ne fait pas encore référence à un comportement culturel spécifique. Les éléments d'une explication sont d'ores et déjà présents sans que l'imputation ethniciste soit effectivement établie. C'est la prise de parole de l'enseignante (E 1) qui va assigner un motif explicatif aux comportements de ces collégiens : II Ah, mais ils agissent de la même façon que lorsqu'ils sont chez eux ! C'est dans leur culture. Les filles sont au service des garçons 11 . La catégorie de collégien passe alors au 
second plan pour donner la priorité à une attribution identitaire générale qui dépasse de loin le seul fait d'être un élève. C'est en effet l'ensemble de la catégorie "garçon" qui se trouve visé dans les propos de E 1. L'affirmation " C'est dans leur culture " confirme qu'il ne s'agit pas de comportements d'adolescents/élèves dont l'attitude idiosyncrasique serait condamnable, mais d'une caractéristique ethnoculturelle déviante inscrite dans les rapports de genre filles/garçons.

À travers ce contournement fondé sur le thème de l'inégalité fille/ garçon, c'est directement l'ethnicisation des relations socioscolaires qui se trouve convoquée. Le thème de la citoyenneté permet de pointer l'altérité déviante d'une catégorie d'élèves dès lors montrée comme se situant en dehors de la "civilisation des mœurs" telle que conçue par les acteurs scolaires de cette réunion.

Cette altérité déviante ne cesse ensuite d'être documentée par les différents interlocuteurs. À telle enseigne qu'une autre enseignante (E 2) fait à son tour allusion à un autre élève en le citant nommément. L'attitude d'Abdoul Jabar envers les filles illustre et conforte les propos précédents. II s'agit donc bien d'un comportement intelligible à partir d'une grille interprétative strictement culturaliste, c'est-à-dire qui donne à entendre les faits et gestes des élèves garçons comme étant diagnosticables sur la base de la détermination d'une appartenance ethnoculturelle dont les pratiques ordinaires sont à la fois violentes et déviantes vis-à-vis du sexe opposé. Il s'agit par ailleurs d'une domination sans faille à laquelle les jeunes filles se soumettent sans aucun droit de résistance, ce que semble confirmer les remarques de la documentaliste et de l'enseignante E 3 : "Mais celui qui a donné un coup de pied à sa sœur, c'est absolument incroyable ! Et la jeune fille, elle n'a rien dit " [documentaliste] ; "Eh oui, ils ont tous les droits $1 /[\mathrm{E} 3]$.

La violence décrite ici relève d'un fait structurel inscrit dans la logique des relations familiales entre frères et sœurs. C'est l'ensemble d'un groupe socioculturel collectivisé de façon hétéronome qui se voit pointé du doigt et affublé de stéréotypes stigmatisants en référence à une lecture de la domination des garçons sur les filles dans un cadre culturel irrespectueux de l'égalité homme/femme. 


\section{- La déviance comportementale généralisée du garçon "arabo- musulman"3}

À ce stade de la discussion, la documentaliste, après avoir cerné la question sous l'angle de l'opposition fille/garçon à l'aune de la différence culturelle, élargit son propos à l'ensemble des pratiques comportementales des élèves en question. Selon la remarque de la documentaliste " Mais il y a tellement de choses à corriger "I, il s'agit véritablement d'un travail de redressement qu'il faudrait exercer sur ce type d'élèves. Ainsi s'opère-til un glissement insensible de la violence subie par les filles à la description de comportements anomiques extraits de la vie ordinaire du collège. Est mentionnée l'attitude d'élèves impénitents refusant de récupérer leurs carnets de correspondance auprès des acteurs scolaires autrement qu'en les "arrachant des mains " ${ }^{4}$. À la violence envers les filles s'ajoute cette espèce de sauvagerie latente qui se manifeste dans leur manière de se saisir de leur carnet de correspondance. Cette sauvagerie semble irrépressible, les acteurs du système éducationnel insistant sur le caractère immuable du comportement de ces élèves: "I On a beau leur dire : "Doucement, prenez les carnets délicatement" ; ça ne change rien " [documentaliste].

La procédure de généralisation est à l'œuvre à travers l'usage du pronom personnel "ils". Pour la suite de la discussion, le procédé consistant à passer insensiblement de la généralisation au cas particulier, et viceversa, n'a plus véritablement lieu d'être. Les locutrices savent de quel type d'élève il est question. Toutes les activités répréhensibles décrites par les acteurs scolaires font référence au type d'élève évoqué dans les premiers échanges. II s'agit bien de l'élève-garçon-arabo-musulman dont les différents comportements énoncés au fil de la conversation servent à nourrir réflexivement la déviance culturellement déterminée. S'établit ainsi une équivalence entre son identité d'élève de telle origine, la violence de ses comportements et sa sauvagerie sous-jacente.

L'enseignante E 5 noircit le tableau déjà sombre de ces élèves: "IIs crachent dans les plateaux II. La documentaliste, par un effet de surprise, met alors l'accent sur la déviance inadmissible et incompréhensible de ces élèves aux yeux des locutrices en utilisant des expressions telles que " C'est

3. L'expression "garçon 'arabo-musulman'" est inspirée du titre de l'ouvrage de GUÉNIF-SOUILAMAS, Nacira ; MACÉ, Éric, Les féministes et le garçon arabe, La Tour-d'Aigues : Éd. de L’Aube, 2004, $106 \mathrm{p}$.

4. Suivant la grammaire catégorielle analysée par Harvey Sacks, il est possible d'entendre un enchaînement de catégories déviantes sous le registre de la brutalité des auteurs de ce type de comportement. Voir SACKS, Harvey, "An initial investigation of the usability of conversational data for doing sociology", in : SUDNOW, David (Ed.), Studies in social interaction, New York : Free Press, 1972, pp. 31-63. 
absolument incroyable " ou encore "Ils crachent dans les plateaux ! C'est pas possible ! II. L'étonnement participe de cette description de la différence ethnoculturelle des collégiens et de la radicalité des oppositions entre un "nous" civilisé et un "eux" sauvage 5 .

Cette opposition se trouve confortée par la suite des échanges. Après avoir souligné la manière dont ces collégiens ne peuvent s'exprimer autrement qu'en hurlant, l'enseignante E 5 en apporte une explication qui semble aller de soi : les élèves ne font que reproduire au collège des pratiques de communication usuelles dans le cadre de leur famille. S'oppose une dualité entre le normal et l'anomique qui dépasse la singularité de tel ou tel élève pour recouvrir une attitude culturelle partagée par l'ensemble d'un groupe. Transférant dans le cadre scolaire des pratiques supposées naturelles au sein des familles, ce groupe finit par agresser les acteurs scolaires, selon les termes de E 5. C'est ici le registre de la violence et de la brutalité intrinsèque au collectif ${ }^{6}$ en question qui se trouve validé par la manière dont ses membres, notamment les garçons, usent de leur voix pour agresser les adultes du collège ou terroriser les jeunes filles qui, effrayées par la présence des garçons, " se mettent à hurler $\|^{7}$.

La domination masculine exercée sur les collégiennes n'est autre que la continuation de pratiques violentes partagées par les garçons arabomusulmans au sein de leur famille. La violence décrite ici concerne l'organisation ethnoculturelle d'un groupe essentialisé. La généralisation de sa déviance implique, de la part des professionnels de l'éducation, que l'objectif d'un apprentissage de la citoyenneté soit de corriger la série de dysfonctionnements énumérés au cours de la discussion.

5. La structure qui régit l'opposition ethniciste entre un "eux" minoritaire et un "nous" majoritaire est analysée par POUTIGNAT, Philippe ; STREIFF-FÉNART, Jocelyne, Théories de l'ethnicité, Paris : Presses universitaires de France, 1995, pp. 9-201. Voir aussi LORCERIE, Françoise (sous la direction de), L'école et le défi ethnique. Éducation et intégration, Issy-les-Moulineaux : ESF Éditeur ; Paris : INRP, 2003, 333 p. (cf. notamment pp. 27-33).

6. Sur certaines modalités de la constitution d'un collectif ethnicisé, on pourra se reporter aux écrits de Jean-Pierre Zirotti, et notamment ZIROTTI, Jean-Pierre, "Jeunes Maghrébins : un groupe sociologiquement pertinent ?”, Cahiers du CERCOM, n 6, juin 1991, pp. 185-197 ; ZIROTTI, JeanPierre, "De l'expérience de la discrimination à la délégitimation. Les jugements des élèves issus de l'immigration sur les décisions d'orientation scolaire et les conditions de leur scolarisation", Cahiers de l'URMIS, $\mathrm{n}^{\circ}$ 10-11, décembre 2006, pp. 101-110.

7. «Et puis on voit la crainte des filles. Dès qu'un garçon les approche, dans les couloirs, elles se mettent à hurler, mais c'est un réflexe. C'est comme un réflexe, mais elles hurlent " [documentaliste]. 


\section{- La revendication d'une "nationalité" comme symptôme de déviance}

La suite de la discussion entraîne les locuteurs sur un terrain qui consiste à rabattre la notion de citoyenneté sur celle de nationalité. La confusion entre ces deux notions ainsi que ses implications normatives apparaissent pleinement à la façon dont l'enseignante E 2 souligne l'affirmation selon laquelle les élèves ne répondraient pas systématiquement "I am French " à la question "I Of what nationality are you ? "I. Visiblement, le fait de ne pas se dire "Français" traduirait un déficit d"'intégration" à "la" société dans laquelle vivent ces élèves. Dire que l'on est "I tunisien ") ou " marocain " semble faire problème pour $E$ 2. Le statut de collégien est alors entaché par l'expression d'une origine nationale incompatible avec la position d'élève. Exprimer l'appartenance à une de ces nationalités expliquerait en partie la déviance des élèves et rendrait compte des problèmes que rencontrent les enseignants avec ce type de collégiens. Ce lien indiscuté entre citoyenneté, nationalité et trajectoire scolaire négative des élèves traduit l'expression particulière d'une relation entre ethnicité et déviance.

À travers leurs propos, les acteurs scolaires établissent une relation évidente entre intégration scolaire, expression assumée d'une nationalité française par les élèves ${ }^{8}$, adhésion aux valeurs associées à l'État-nation et citoyenneté. De sorte que le duo citoyenneté française et nationalité étrangère semble une hérésie normative, conduisant à l'impossibilité d'une éventuelle double appartenance qui ne soit dysfonctionnelle. Les difficultés rencontrées au sein de l'école sont renvoyées à des pratiques culturelles, voire à des sentiments d'appartenance qui trahissent le respect des valeurs de l'école républicaine. On a là un effet de fixation et de dichotomisation des "identités" qui participe de leur essentialisation.

Les difficultés rencontrées par les acteurs scolaires avec certains élèves sont en partie imputées à l'organisation structurale des rapports de genre au sein des familles dites arabes : II Mais ils viennent de familles arabes et ils continuent à garder les mêmes manières de penser. Dans les familles, chez elles, les femmes sont dans leur coin, les hommes dans un autre. II y a toujours ce même clivage "I [E 5]. Ici, l'usage du qualificatif "arabe", pris dans l'argument de $E 5$, ne semble pas établir de différence entre les diverses origines (tunisienne, marocaine, etc., ni entre "arabe" et

8. Pour une description de la relation entre État-nation, ethnicité et intégration, on pourra se reporter aux analyses de Françoise Lorcerie. Voir notamment LORCERIE, Françoise, "Le paradigme de l'ethnicité", in : LORCERIE, Françoise (sous la direction de), L'école et le défi ethnique. Éducation et intégration, Issy-les-Moulineaux : ESF Éditeur ; Paris : INRP, 2003, pp. 19-98 (cf. notamment pp. 53-98). 
"berbère"), tandis que précédemment le locuteur E 7 mentionnait d'un air déconcerté la manière dont les élèves confondaient nationalité et origine "arabe" : II J'irai encore plus loin, ils ne disent pas "Tunisien", ils disent "Arabe". Ils ont déjà du mal à faire la différence "I.

Selon le contexte d'énonciation, la différenciation nationale peut ou non être de mise. Toujours est-il que lorsqu'il est question de donner sens à certaines situations difficiles à gérer par les acteurs scolaires, tout semble indiquer que le terme générique "arabe" suffit à argumenter dans le sens d'une altérité ethnoculturelle déviante.

\section{La pathologisation des difficultés des élèves}

Face aux difficultés que manifestent les élèves, il est cependant des situations où leur altérité ethnoculturelle déviante n'est pas directement mise en cause. D'autres considérations, d'ordre psychologique ou médical, peuvent servir à argumenter la perte de contrôle de la situation par les acteurs scolaires et à envisager en conséquence des solutions adaptées.

La séquence transcrite ci-dessous est extraite d'un Conseil de zone d'éducation prioritaire au cours duquel les acteurs de l'éducation se réunirent autour du thème suivant : " Comportements psycho-affectifs des élèves, résolution des problèmes de troubles psychologiques, voire psychiatriques. Et personnes qualifiées pour intervenir 11 . Les membres vont débattre, non sans quelques désaccords, sur la manière de qualifier les difficultés scolaires que manifestent les collégiens et sur les solutions à y apporter.

E 1 : enseignant de sciences de la vie et de la terre (SVT)

IEN : inspectrice de l'Éducation nationale

Psychologue scolaire

E 2 : enseignant d'histoire-géographie

E 3 : enseignante d'anglais

E 4 : enseignante d'espagnol en classes de $4^{\text {e }}$, de $3^{\text {e }}$ et ponctuellement en classe de Section d'enseignement général et professionnel adapté (SEGPA) ${ }^{9}$.

9. La SEGPA est destinée aux élèves « présentant des difficultés scolaires graves et durables [et qui] ne maîtrisent pas toutes les connaissances et compétences attendues à la fin de l'école primaire, en particulier au regard des éléments du socle commun", http://eduscol.education.fr/D0081/ segpa.htm\#2 
E1 : Bien, je voudrais dans un premier temps m'expliquer sur le choix du thème de cet atelier. Donc, j'ai désiré que nous discutions sur ce sujet car je remarque qu'il nous arrive au collège des élèves de plus en plus déstructurés. Des élèves qui n'arrivent pas à suivre en classe, je ne parle même pas de faire le programme, mais simplement de rester assis une heure de temps. Moi, je pense qu'il faudrait un suivi médical, voire psychiatrique pour certains de nos élèves.

[L'inspectrice de l'Éducation nationale prend la parole pour contester le profil psychiatrique des élèves que vient de dresser E 1. Puis, la psychologue scolaire prend la parole pour renforcer les propos de l'inspectrice].

Psychologue scolaire: Mais attendez, Monsieur. Que les élèves aient des troubles psychologiques, des difficultés qui empêchent leur apprentissage, je suis tout à fait d'accord, mais ces élèves n'ont pas besoin pour autant d'un suivi psychiatrique. Cela fait longtemps que nous en sommes revenus de ces confusions sur des situations qui n'ont absolument rien à voir.

E 1 : Bien, alors si vous ne voulez pas parler de psychiatrie, parlons de troubles psychologiques, d'accord. Mais nous, face à de telles situations, que pouvonsnous faire? Vous savez, il y a des périodes ici où ce n'est franchement pas gai. Je peux vous dire que durant la guerre du Golfe, en 91, c'était particulièrement tendu. II était devenu impossible de les tenir. Alors, face à de telles situations de déstructuration psychique, intellectuelle et affective, que pouvons-nous faire?

Et puis il y a un autre problème. C'est que ce sont des enfants pour qui la communication est cassée. Ils ne savent plus communiquer avec des mots. Ce sont des jeunes qui parlent avec un langage qui prend souvent la forme de bruits et d'onomatopées. II n'est plus possible de leur parler. Et ça, je pense que ça doit venir de leur relation aux parents, je pense que ce sont des enfants qui ne doivent pas communiquer avec leurs parents. Ce sont des enfants qui chez eux, lorsqu'ils rentrent, se mettent devant la télé et n'ont pas de conversations. Leurs échanges sont minimaux, alors bien sûr, ici nous les embêtons. Parfois, j'ai le sentiment d'être inhumain à leur égard. II ne faudrait pas les enfermer dans une salle, il faudrait tout le temps être dehors avec eux ; je le vois lorsque i'ai cours l'après-midi, ils ont été présents toute la matinée, la seconde partie de la journée leur est véritablement un calvaire. Mais j'ai le sentiment qu'ils sont venus parce qu'il faut être présent à l'école, mais qu'ils étaient chez eux à regarder la télé, et que moi, je les ai dérangés dans leur programme. Alors quand on a des difficultés avec un élève, le temps que l'on fasse chercher le $\mathrm{CPE}^{10}$ ou que monte le surveillant, la situation s'est envenimée, et c'est déjà trop tard.

E 2 : En effet, il faudrait un système de sonnette d'alarme que l'on actionnerait dès que l'on sent venir le problème, de manière à prévenir la situation

10. Conseiller principal d'éducation. 
et que l'on puisse d'emblée transmettre le problème à une personne spécialisée dans la question.

E 3 : Et cela s'explique en partie parce que nous avons affaire à des élèves avec qui, justement, nous ne communiquons pas, ou qui ne parlent pas français, et qui par conséquent ont des problèmes de compréhension et d'accession à la signification de ce qu'on essaie de leur dire. Pour certains, il y a de vrais problèmes de latéralité, des problèmes de repères dans l'espace et dans le temps qu'il faut absolument corriger. Et plus ça va, plus la situation s'aggrave, et on voit des élèves passer de classe en classe sans savoir lire ou écrire. Bientôt nous aurons des élèves de troisième dans des situations semblables à celles des élèves de SEGPA.

E 4 : Oh, vous savez, moi je donne des cours d'initiation à l'espagnol en SEGPA, vous avez des élèves qui ne sont pas bêtes du tout, et avec lesquels on ne rencontre pas les mêmes problèmes de discipline qu'avec ceux du collège. Étant en petit nombre et ayant une équipe pédagogique qui se concerte et des enseignants fixes pour chaque classe, avec pratiquement aucun changement de salle et de va-et-vient dans les couloirs, vous avez des élèves très bien encadrés et qui n'ont pas cette agression vis-à-vis des enseignants comme au collège.

E 1 : Madame l'Inspectrice, vous savez, au collège, nous avons des élèves qui sont franchement limite, et je me demande si la différence avec la SEGPA est encore perceptible.

E 3 : Vous avez la sixième F, qui est une sixième dite "de consolidation", mais qui finalement fonctionne comme une sixième normale, c'est-à-dire avec un effectif aussi important, ou presque : une quinzaine d'élèves qui n'ont rien à faire en sixième. Ils ne savent ni lire ni écrire ; ils auraient dû, pour certains d'entre eux, redoubler leur cours moyen car vu leur âge, ils le pouvaient. Je ne sais pas ce que font les enseignants du primaire ; ils doivent être dépassés par cette situation. Aussi, ils nous envoient au collège tous les élèves arrivés en fin de cursus. D'autres auraient dû aller en SEGPA, mais comme leurs parents et les élèves ne veulent pas $y$ aller, et que nous ne pouvons pas les y obliger, ils nous les envoient en sixième.

Dans cette classe, ils devraient tous être en SEGPA. [lls] n'ont rien à faire en sixième. De ce fait ils sont complètement perdus et nous ne pouvons les aider individuellement car ils sont trop nombreux. Et du reste, ce sont des cas qui ne relèvent plus de notre compétence. 


\section{- Une divergence : problèmes psychiatriques ou troubles psychologiques?}

Par rapport à la séquence analysée précédemment, la question culturelle ne constitue pas l'argument prévalent. Ici, ce sont des caractéristiques médicopsychologiques, voire psychiatriques, appliquées habituellement à des individualités, qui permettent de généraliser une situation. II semblerait que la ZEP soit un lieu qui regroupe un nombre non négligeable d'élèves porteurs de caractéristiques psychopathologiques incompatibles avec une participation scolaire ordinaire. Quand bien même le locuteur $E 1$ n'opère pas une généralisation systématique, le phénomène semble suffisamment remarquable et embarrassant pour justifier d'un débat dans l'instance délibérative de la ZEP.

La psychologue scolaire ne partage pas le point de vue de E 1. Elle prend la parole pour établir une distinction entre le II suivi médical, voire psychiatrique "I réclamé par son collègue et la reconnaissance de "I troubles psychologiques, [de] difficultés qui empêchent leur apprentissage ". La locutrice déplore une confusion entre des domaines qui " n'ont absolument rien à voir "I. La correction qu'elle apporte ne consiste pas tant à contester l'intervention de E 1 qu'à recadrer la discussion afin de s'entendre sur les mots. E 1 s'accorde alors avec la psychologue pour dire qu'il existe bien des profils scolaires perturbés par des troubles d'ordre psychologique. S'observe ainsi une démarche comparable à celle mentionnée dans l'analyse de la première séquence : les difficultés manifestées par les élèves et auxquelles doivent faire face les acteurs scolaires sont renvoyées à des schémas explicatifs qui ne remettent pas en cause les pratiques des professionnels ou l'organisation interne du collège.

En revanche, on peut noter l'importance des propos de l'enseignante d'espagnol (E 4) qui rappellent les difficiles conditions de travail auxquelles l'équipe pédagogique doit faire face : ॥ En SEGPA [...] on ne rencontre pas les mêmes problèmes de discipline qu'avec ceux du collège. Étant en petit nombre et ayant une équipe pédagogique qui se concerte [...] vous avez des élèves très bien encadrés et qui n'ont pas cette agression vis-à-vis des enseignants comme au collège 11 . Ces conditions détériorées d'exercice de la fonction renvoient à une politique éducative dans les ZEP qui, sous couvert officiel d'action corrective des inégalités scolaires, maintient en réalité des situations d'apprentissage fortement injustes : classes surchargées, enseignants éreintés par un travail qui ne laisse plus de temps pour la concertation pédagogique, encadrement des élèves forcément réduit. La dégradation de ces conditions de travail se traduit par un regain d'agressivité de certains élèves vis-à-vis des enseignants et un rejet de l'institution sco- 
laire. Faisant les frais de cette détérioration des conditions d'apprentissage, ces élèves développent parfois des comportements jugés "violents" qui confortent le point de vue des acteurs éducatifs... Cercle infernal qui nourrit les divers processus de catégorisation ethnique.

\section{- Troubles psychologiques : une catégorie fourre-tout de l'altérité}

Consentant à requalifier la question psychiatrique en troubles psychologiques, E 1 détaille son argumentation en faisant référence à un événement qui appartient, au moment de l'énonciation, à une situation ancienne. La "première" guerre du Golfe, à laquelle il est fait allusion, date de 1991, c'est-à-dire une dizaine d'années plus tôt. Le rapport entre les troubles psychologiques évoqués et le conflit irakien est pour le moins énigmatique, sinon qu'il s'agit d'un "haut fait", d'un récit qui fait repère pour le collectif du collège. Les élèves auraient pris parti pour les ennemis de la coalition internationale d'alors et auraient vécu, par soldats interposés, les événements à partir de leur statut de collégiens. On voit que le lien entre des troubles psychologiques, la guerre et les élèves relève d'une construction interprétative ethniciste qui participe d'une lecture pathologisante. Cette interprétation assimile l'identification supposée ou réelle des élèves avec Saddam Hussein à des II situations de déstructuration psychique, intellectuelle et affective II. La référence à un événement spécifié dans le temps et dans l'espace autorise la construction ex post d'une identité ethnoculturelle déviante, dont les frontières avec le pathologique sont poreuses.

Contrairement à la première séquence, où les élèves hurlaient, dans cette seconde séquence l'origine ethnoculturelle des collégiens n'est pas directement mise en cause. II s'agit plutôt d'établir ici un couplage entre des troubles psychologiques, le comportement déstructuré des élèves lors du conflit irakien et la "cassure" de la communication chez les élèves (c'est l'énigmatique expression : " des enfants pour qui la communication est cassée 11). Cependant, dans les deux séquences, loin de se contredire, les arguments se complètent — sauvagerie dans un cas, régression psychoaffective dans l'autre - et attestent de l'altérité des élèves ainsi que de leurs familles. 


\section{- L'adaptation aux difficultés des élèves}

Les pratiques ethnoculturelles déviantes des élèves conduisent, dans les propos tenus par E 1, à conclure à un clivage net et à une incompatibilité radicale entre les comportements carencés des familles et les pratiques scolaires attendues: " Alors bien sûr, ici nous les embêtons. J'ai le sentiment qu'ils sont venus parce qu'il faut être présents à l'école, mais qu'ils étaient chez eux à regarder la télé, et que moi, je les ai dérangés dans leur programme 11.

Dans cette citation, l'élève déviant, transformé en input dysfonctionnant, vient troubler un ordre scolaire pour lequel il n'est définitivement pas préparé à agir convenablement. C'est pour pallier cette inadaptation que $\mathrm{E} 2$ propose un scénario fonctionnel de prévention de la déviance scolaire : "In effet, il faudrait un système de sonnette d'alarme que l'on actionnerait dès que l'on sent venir le problème, de manière à prévenir la situation et que l'on puisse d'emblée transmettre le problème à une personne spécialisée dans la question 11.

L'argumentation développée successivement par E 1 et $\mathrm{E} 2$ débouche sur un protocole d'adaptation/prévention qui s'inscrit dans une logique discursive semblant détruire la contingence inhérente à l'activité pédagogique. Celle-ci se voit réduite à un acte idéalisé entre des professionnels théoriques et des élèves engagés dans un processus d'apprentissage rationalisé à l'extrême. C'est en raison de cette vision policée de la relation pédagogique que toute potentialité d'acte d'indiscipline doit être neutralisée ex ante par un dispositif préventif à l'instar du panoptique de Bentham qui inspira Foucault dans sa réflexion sur la généalogie des "disciplines".

Réclamer un système de "I sonnette d'alarme " afin de " transmettre le problème à une personne spécialisée dans la question " revient à reconsidérer la division du travail scolaire. La transmission des savoirs relèverait de la compétence de l'enseignant et l'activité de prévention disciplinaire serait déléguée à d'autres adultes qui interviendraient sur demande dans la salle de cours. Paradoxalement, plus le travail de socialisation des élèves au monde scolaire fait partie intégrante de l'activité d'enseignement, plus les enseignants l'évitent et souhaitent le déléguer à des "spécialistes". 


\section{- Filières générales et SEGPA : la confusion des genres ?}

S'organise insensiblement un travail d'évaluation d'une déviance fondée sur l'appartenance ethnoculturelle des élèves qui aboutit à une dévalorisation de facto de leurs capacités à réussir un parcours scolaire traditionnel. Ce raisonnement conduit à fragiliser l'apprentissage d'un savoir partagé par l'ensemble des collégiens d'un même niveau, et donc à affaiblir effectivement leur compétence dans ce domaine. Peut-être est-ce l'emballement de ce phénomène qu'expriment les acteurs scolaires, comme l'indique ici E 3 : " Et plus ça va, plus la situation s'aggrave, et on voit des élèves passer de classe en classe sans savoir lire ou écrire. Bientôt nous aurons des élèves de troisième dans des situations semblables à celles des élèves de SEGPA 11.

La séparation entre le disciplinaire et l'enseignement des savoirs revendiquée par certains professionnels conduit à construire des profils socioscolaires d'élèves toujours plus inadaptés au cadre éducatif. Un nombre accru d'entre eux finit par se ressembler quelle que soit la filière ou la section dans laquelle ils sont orientés. Leur inadaptation générale fait corps avec leur altérité dès lors décrite comme déviante et indistinctement dévalorisée. La structure éducative fonctionne par l'absurde. Plus le versant correcteur et répressif est mis en avant, plus le constat est fait d'une homogénéisation des niveaux avec des élèves qui ne sont par définition jamais à leur place (E 3 : " Ce sont des cas qui ne relèvent plus de notre compétence 11$)$, quelles que soient les orientations qui leur sont proposées. C'est alors la raison d'être même de la ZEP qui se trouve fragilisée...

Ces observations conduites dans une ZEP amènent à penser que les expériences éducatives se trouvent progressivement perverties par la réification des appartenances individuelles et collectives ${ }^{11}$. Ce type d'essentialisation négative et de naturalisation des appartenances ethnoculturelles n'est pas favorable à l'atténuation des situations conflictuelles que l'on trouve dans ces espaces urbains ${ }^{12}$.

11. Cf. HONNETH, Axel, La réification : petit traité de théorie critique, Paris : Éd. Gallimard, 2007, 141 p. Selon Axel Honneth, le processus de réification conduit à accorder le primat à la connaissance objectivée des individus catégorisés sur leur reconnaissance subjective en tant que singularité existentielle dont les spécificités ne sauraient être renvoyées à des appartenances collectives hypostasiées.

12. Sur la délégitimation des cadres institutionnels et la réappropriation combative des catégories ethnoculturelles par les acteurs minorisés, voir ZIROTTI, Jean-Pierre, "Comment les cadres de légitimation des pratiques productrices d'humiliation servent de ressource à leur critique ? Genèse d'acteurs sociaux critiques : l'exemple des élèves maghrébins en France", in : GUILLAUD, Yan ; WIDMER, Jean (sous la direction de), Le juste et l'injuste. Émotions, reconnaissance et actions collectives, Paris : Éd. L'Harmattan, 2009, pp. 77-95. 